THERMAL SPIKE MODEL OF ION-INDUCED GRAIN GROWTH*

\author{
D. E. Alexander** and G. S. Was*** \\ **Materials Science Division \\ Argonne National Laboratory \\ Argonne, IL 60439 \\ ***Department of Nuclear Engineering \\ CONF-901105--77 \\ DE9 1006571 \\ University of Michigan, Ann Arbor, MI 48109
}

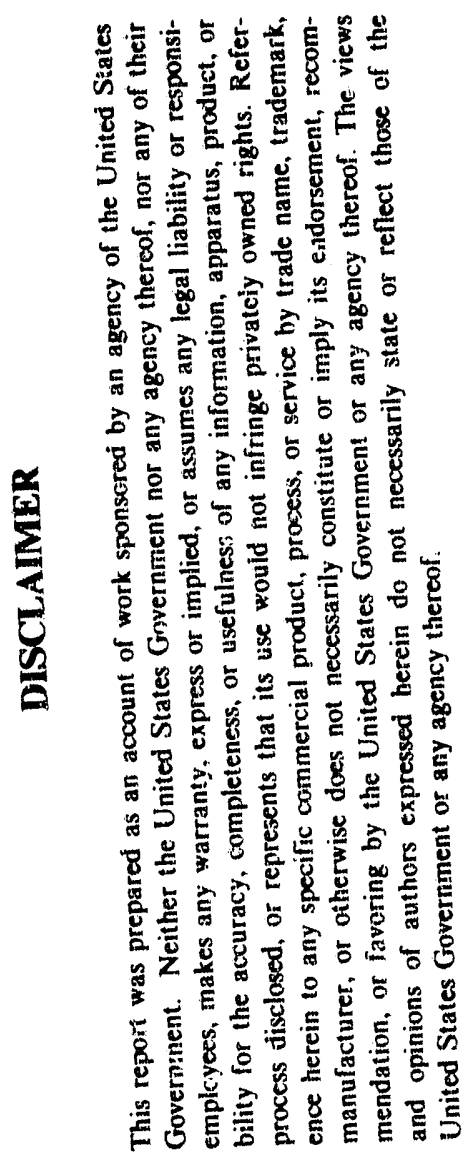

November 1990

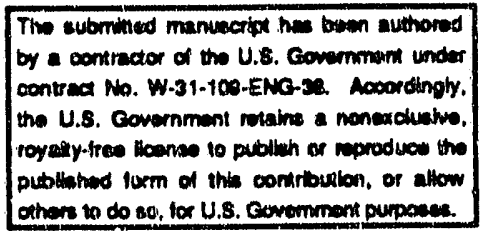

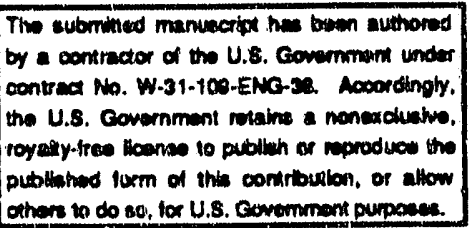

MASItK

Submitted to the Materials Research Society 1990 Fall Meeting, Nov. 26-Dec. 1, 1990 , Boston, MA.

*This work was funded under DOE-BES contract \#W-31-109-ENG-38 (D. Alexander) and MISF grants \#DMR8603174 and \#DMR8903138 (G. Was). 


\section{THERMAL SPIKE MODEL OF ION-INDUCED GRAIN GROWTH}

\section{DALE E. ALEXANDER* AND GARY S. WAS**}

* Materials Science Division, Argonne National Laboratory, Argonne, IL 60439

**Department of Nuclear Engincering, University of Michigan, Ann Arbor, MI 48109

\section{ABSTRACT}

A thermal spike model has been developed to describe the phenomenon of ion iradiationinduced grain growth in metal alloy thin films. In single phase films where the driving force for grain growth is the reduction of grain boundary curvature, the model shows that ion-induced grain boundary mobility, $M_{\text {ion }}$, is proportional to the quantity $F_{D}^{2} / \Delta H_{c o h}^{3}$, where $F_{D}$ is the deposited ion damage energy and $\Delta \mathrm{H}_{\text {coh }}$ is the cohesive energy of the element or alloy. Experimental grain growth results from ion irradiated coevaporated binary alloy films compare favorably with model predictions.

\section{INTRODUCTION}

Heavy ion irradiation has been observed to induce nearly athermal normal grain growth in initially fine grained $(<100 \AA)$ thin films. Because of the importance of grain size in a number of thin film properties (e.g. electromigration characteristics), several studies [1-5] have been undertaken to investigate ion-induced grain growth. Despite these studies, a complete understanding of the phenomenon is lacking.

Previously, Atwater et al. [2] proposed a transition state model for describing ioninduced grain growth. The grain growth rate was determined to follow a first order dependence on the point defect generation per ion or likewise on the deposited damage energy per ion, $F_{D}$. While the model was successful in describing observations of ion induced grain growth in $\mathrm{Au}$, $\mathrm{Si}$ and $\mathrm{Ge}$ thin films [2], more receni results suggest that collisional displacements alone are inadequate for describing ion-induced grain growth. Li et al. [3] observed large differences in grain growth among irradiated Pt and Au films, despite the fact that their similar masses and displacement energies suggested that they should behave collisionally the same. Liu [5] and Alexander et al. [1] observed variations in grain growth rates that scaled with the cohesive energy, $\Delta \mathrm{H}_{\text {ooht }}$ of the targets, indicating that material parameters in addition to collisional related material properties are also important. Results such as these led Liu et al. [3] to suggest that ioninduced grain growth is a thermal spike phenomenon.

Thermal spikes occur in the final phase of an irradiation-induced displacement cascade [6]. When a heavy ion penetrates a solid, displacement of lattice atoms occurs. These displaced atoms, or recoils, in turn cause further displacements inducing a cascade. The collisional phase of the cascade ends when the ion or recoils no longer possess sufficient energy to cause further dispiacements. This is followed by a thermal spike phase where the remaining kinetic energy is thermalized in the lattice. As a result of this thermalization, each primary recoil causes a 
temperature spike to occur in a small localized region of the lattice. Due to this heating, additional defects are produced and are stimulated to migrate. However, unlike the high energy collisional phase, the average kinetic energy during the thermal spike phase is low ( $\mathrm{l} \mathrm{eV})$ and hence atom migration is strongly affected by material related thermochemical quantities (e.g. $\Delta \mathrm{H}_{\text {cob }}$ ) which are of the same magnitude.

As will be shown, atom jumps induced by thermal spikes can be used to describe the macroscopic phenomenon of ion-induced grain growth. If the spikes occur on, or near a grain boundary, atoms can be thermally activated to jump across the boundary. If a net number of atom jumps occur in one direction across the boundary, the boundary migrates in the opposite direction. In this paper, we outline the development of a thermal spike model of ion-induced grain growth, the complete details of which are presented elsewhere [7]. The results are then applied to experimental observations of ion-induced grain growth in coevaporated alloy films.

\section{MODEL DEVELOPMENT}

The connection between the microscopic effects of thermal spikes and the macroscopic grain growth phenomenon is made by derivation of the thermal spike parameter, $\eta$, which is the net number atom jumps induced across a grain boundary by a thermal spike, per unit length of spike. Once $\eta$ is determined, it can be related to macroscopic grain boundary migration by the following argument. After a dose, $\Phi$, of ions the total number of jumps induced by thermal spikes per unit volume of material is $\eta \Phi$. The net number of jumps per atom across the boundary is $\eta \Phi / \rho$. These atom jumps displace the boundary by a distance $\delta$, where $\delta$ is equal to the boundary width. After an increment of dose $\Delta \Phi$, the distance a grain boundary migrates is $\Delta \mathrm{l}=\eta \delta \Delta \Phi / \rho$. Therefore the grain growth rate per unit time for constant dose rate, $\Phi$, is,

$$
\frac{\mathrm{dL}}{\mathrm{dt}}=\frac{\eta \dot{\delta} \dot{\phi}}{\rho}
$$

The objective is thus to derive the parameter $\eta$. The approach taken parallels that of Vineyard [8] in which atomic motion-induced by thermal spikes of cylindrical and spherical geometry were examined. Analysis for the present study was restricted to cylindrical spikes which are expected to result from heavy ion irradiation in dense materials.

A cylindrical thermal spike is induced from the energy deposited by an ion or recoil penetrating a crystalline lattice. The ion or recoil deposits energy in the lattice at a constant specified linear energy loss, $\varepsilon$, (energy per unit length) and the subsequent dissipation of this energy results in the creation of a temperature distribution in a small localized cylindrical volume. An analytical expression for the temperature distribution, $T(s, t)$, resulting from the energy deposition can be obtained by solving the heat conduction equation, in cylindrical geometry, considering at time $t=0$ an initial distribution of deposited energy as $\varepsilon \delta^{2}(s)$ where $\delta^{2}(s)$ is the two-dimensional delta function. Vineyard [8] solved this equation by assuming a simple power 
law dependence for the thermal conductivity, $x$, and heat capacity, $c$, on temperature such that $k=K_{0} T^{p-1}$ and $c=c_{0} T^{p-1}(p \geq 1)$. The solution obtained was,

$$
T(s, t)=\left(\frac{p \varepsilon}{4 \pi \kappa_{0} t}\right)^{1 / p} e^{-\frac{c_{0} s^{2}}{4 p \kappa_{0} t}}
$$

where $s$ is the radial distance from the cylinder axis, $t$ is the time, $\kappa_{0}$ and $c_{0}$ are the ambient values of the thermal conductivity and the heat capacity, respectively.

We now identify $R(s, t)$ as a thermally activated net atomic jump rate across a grain boundary per unit volume. $R(s, t)$ is a function of the temperature distribution given in eqn.(2) (i.e. $R(s, t)=f[T(s, t)])$. Once $R(s, t)$ is determined, $\eta$ is found by integrating over time and space:

$$
\eta=\int_{0}^{\infty} 2 \pi s d s \int_{0}^{\infty} R(s, t) d t
$$

An expression for $R(s, t)$ may be developed by considering atoms located at different sites I and $I$, with site $I$ lower in free energy by an amount $\Delta \mu$. In order for atoms to transfer between sites, an energy barrier, $Q$, must be overcome. Since site II is at a slightly lower free energy than site $\mathrm{I}$, a potential gradient $(\mathrm{d} \mu / \mathrm{dx}$ ) exists which may be approximated by $\Delta \mu / \delta$, where $\delta$ is the grain boundary width. Under conditions of thermal agitation, there will be a net transfer of atoms from I to II as a result of the potential gradient. Using a reaction rate argument [9], it can be shown that the net rate of jumps from site I to site II, $v_{\text {net }}$, is,

$$
v_{\text {net }}=k_{0} e^{-Q / k T}\left(1-e^{-\Delta \mu / k T}\right)
$$

where $\mathrm{k}_{0}$ is a frequency factor, $\mathrm{k}$ is Boltzmann's constant and $\mathrm{T}$ is the temperature. In single phase systems, the driving force for normal grain growth is assumed to be the reduction of grain boundary curvature [10] in which case the chemical potential difference is given by the GibbsThompson expression, $\Delta \mu=-4 \gamma \Omega / \mathrm{L}$, where $\mathrm{L}$ is the average grain diameter, $\gamma$ is the grain boundary surface energy and $\Omega$ is the atomic volume. Hence, eqn.(4) is further simplified by using this expression for $\Delta \mu$ and assuming that $\Delta \mu<<k T$ yielding,

$$
R_{\text {curv }}=\frac{4 k_{0} \gamma \Omega \rho}{k T L} e^{-Q / k T}
$$

The expression for $\eta_{\text {curv }}$ is found by substituting the expression for $R_{\text {carv }}$, with $T=T(s, t)$, for $R(s, t)$ in eqn $(3)$. The resulting integration yields,

$$
\eta_{\text {curv }}=\frac{k_{0} \gamma \Omega p \varepsilon^{2} p^{3} k^{2 p}}{2 \pi \kappa_{0} c_{0} Q^{2 p+1}} \Gamma(2 p+1)
$$


where $\Gamma(2 p+1)$ is the gamma function with argument $2 p+1$. Eqn.(6) is further modified by assuming $\varepsilon \approx F_{D}$ where $F_{D}$ is the deposited ion damage energy. Assuming that the activation energy scales with the cohesive energy according to $Q=-\beta \Delta H_{\text {coh }}$, and that $p=1, \eta_{\text {curv }}$ becomes,

$$
\eta_{\text {curv }}=-\frac{k_{0} \gamma \Omega \rho k^{2}}{\pi \kappa_{0} c_{0} L \beta^{3}} \frac{F_{D}^{2}}{\Delta H_{c o h}^{3}} .
$$

The ion-induced grain boundary mobility, $M_{\text {ion }}$, may be found by comtining eqn.(1) with the classical expression for grain boundary migration [9], $\mathrm{dL} / \mathrm{dt}=-\mathrm{M}_{\mathrm{ion}}(\mathrm{d} \mu / \mathrm{dx})$. Using the potential gradient due to grain boundary curvature, $\mathrm{M}_{\text {ion }}$ becomes,

$$
M_{\text {ion }}=-\frac{k_{0} \delta^{2} k^{2} \dot{\Phi}}{4 \pi \kappa_{0} c_{0} \beta^{3}} \frac{F_{D}^{2}}{\Delta H_{c o h}^{3}}
$$

\section{COMPARISON BETWEEN MODEL AND EXPERIMENTAL RESULTS}

The linear dependence of mobility on the parameter $\mathrm{F}_{\mathrm{D}}^{2} / \Delta \mathrm{H}_{\text {coh }}^{3}$, as predicted in eqn.(8), may in part be evaluated by examining grain growth data recently obtained for ion irradiated coevaporated alloy films [1]. Thin films of $\mathrm{A}-20$ at.\%B alloys ( $\mathrm{A}=\mathrm{Au}, \mathrm{Pt}, \mathrm{Ni} ; \mathrm{B}=\mathrm{Co}, \mathrm{Ti}, \mathrm{V}, \mathrm{Ni}, \mathrm{Al}$ ) were irradiated at room temperature using $1.7 \mathrm{MeV}$ Xe ions. Using measured average grain size versus ion dose data, it was possible to derive experimental values $M_{\text {ion }}$ [7].

Fig. 1 shows the variation in $M_{\text {ion }}$ values with the parameter $F_{D}^{2} / \Delta H_{c o h}^{3}$. With the -exception of $\mathrm{Ni}-\mathrm{Al}$, a trend is present vith larger values of $\mathrm{F}_{\mathrm{D}}^{2} / \Delta \mathrm{H}_{\text {coh }}^{3}$ corresponding to larger ion-induced grain boundary mobilities. Since the alloys studied (except Ni-Al) were all collisionally similar (i.e. similar $F_{D}$ and displacement energies), the enhanced grain boundary mobility observed for Au-Co in Fig. 1 is traced directly to its lower value of $\Delta H_{\text {coh. }}$. This translates into a lower activation energy for grain growth and as a result, irradiation-induced thermal spikes more easily induce atom jumps across grain boundaries in this alloy [1].

The low mobility value observed in Fig. 1 for the Ni-Al alloy system is likely due to the presence of a second phase which formed during irradiation. All the binary alloys remained single phase solid solutions during irradiation with the exception of the Ni-Al system. In this alloy, a hexangonal close-packed phase formed in addition to the FCC solid solution phase. The presence of this second phase likely inhibited grain growth resulting in the low nobility value observed for this system.

The thermal spike model predicts a dependence of the grain growth rate on the square of the deposited damage energy, $F_{D}$. This result differs from experimental observations and model predictions of irradiation-induced grain growth by Atwater [2]. However, previous work by Liu [5] supports this functional dependence of $F_{D}$. It is noted however, that the alloys chosen for study in [1] did not vary substantially in values of $F_{D}$, and thus were not best suited for a comp.re evaluation of the effect of the deposited damage energy on grain growth. 


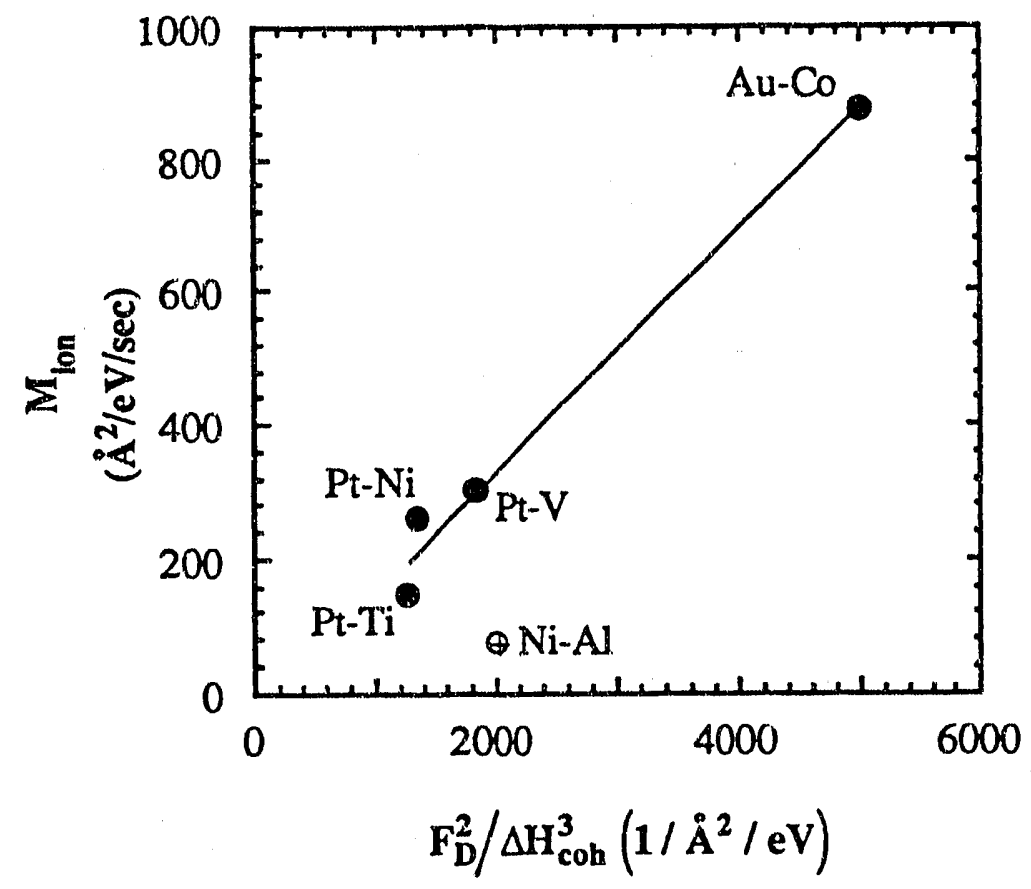

Fig. 1. Variation in experimental $\mathrm{M}_{\text {ion }}$ values with $\mathrm{F}_{\mathrm{D}}^{2} / \Delta \mathrm{H}_{\text {coh }}^{3}$ for coevaporated films irradiated with $1.7 \mathrm{MeV}$ Xe ions. Solid line is a linear least-squared-error fit to the points excluding the $\mathrm{Ni}-\mathrm{Al}$ result.

Table I. Values of constants used in ion-induced grain boundary mobility analysis.

\begin{tabular}{cc}
\hline Constant & Value \\
\hline$k_{0}$ & $3 \times 10^{13} \mathrm{sec}^{-1}$ \\
$\delta$ & $3 \AA$ \\
$\gamma$ & $3 \times 10^{14} \mathrm{eV} \mathrm{cm}^{-2}$ \\
$\Omega$ & $15 \AA^{3}$ \\
$c_{0}$ & $1.3 \times 10^{19} \mathrm{eV} \mathrm{K}^{-1} \mathrm{~cm}^{-3}$ \\
$\kappa_{0}$ & $6.3 \times 10^{18} \mathrm{eV} \mathrm{cm}^{-1} \mathrm{~K}^{-1} \mathrm{sec}^{-1}$ \\
$\dot{\Phi}$ & $1.7 \times 10^{12} \mathrm{~cm}^{-2} \mathrm{sec}^{1}$ \\
$k$ & $8.62 \times 10^{-5} \mathrm{eV} \mathrm{K}^{-1}$ \\
\hline
\end{tabular}


Using the data in Fig. 1, it is possible to estimate the proportionality constant $\beta$, used to scale the activation energy for grain growth with the cohesive energy of the alloy, $Q=-\beta \Delta H_{\text {coh }}$. The slope, $m$, determined from the plot of experimental ion-induced mobilities, $M_{\text {ion }}$, (with the exception of $\mathrm{Ni}-\mathrm{Al}$ ) versus the parameter $\mathrm{F}_{\mathrm{D}}^{2} / \Delta \mathrm{H}_{\text {coh }}^{3}$ shown in Fig. 1 can be used to find $\beta$. From eqn.(8), $\beta$ is expressed as,

$$
\beta=\left(\frac{k_{0} \delta^{2} k^{2} \dot{\Phi}}{4 \pi \kappa_{0} c_{0} m}\right)^{1 / 3}
$$

Values for the constants in the parentheses may be estimated and are given in Table I. Using these values, the proportionality constant for ion-induced grain growth (IYGG) is determined to be $\beta_{\text {IIGG }}=0.06$. This value is less than the constant determined for jon beam mixing (IM) experiments, $\beta_{\mathrm{IM}}=0.14$ [11], in which a similar thermal spike model approach has also been used. The fact that $\beta_{\text {IIGG }}$ is only a factor $\sim 2.3$ different from $\beta_{L V}$ is quite remarkable since the two parameters were obtained from experimental data examining different phenomena (mixing versus grain growth). Furthermore the smaller value of $\beta_{\mathrm{IIGG}}$ is consistent with the idea that atomic diffusion across grain boundaries is easier, having a lower activation energy, than diffusion within the lattice.

\section{SUMMARY}

An ion-induced grain growth model has been developed in which irradiation-induced temperature spikes thermally activate atom jumps which result in boundary migration. For single phase systems, driven by the reduction in grain boundary curvature, the model predicts a linear dependence of the ion-induced boundary mobility on the parameter $F_{D}^{2} / \Delta H_{c o h}^{3}$. Using this thermal spike approach, it is thus possible to account for both irradiation-related material effects $\left(F_{D}\right)$ and non-irradiation material effects $\left(\Delta \mathrm{H}_{\text {coh }}\right)$ on ion-induced grain growth. Hence, as illustrated in a comparison of experimental grain growth results from coevaporated alloy films, this approach provides a framework for understanding variations in grain growth observed among collisionally similar thin film systems. 'Finally, through analysis of data from coevaporated alloy films combined with analytical results of the model, it was possible to determine the value of the proportionality constant :elating the cohesive energy to the activation energy for grain growth. The constant was evaluated to be $\beta_{\mathrm{IIGG}}=0.06$.

\section{ACKNOWLEDGEMENTS}

This work funded under DOE contract \#W-31-109-ENG-38 (D. Alexander) and NSF grants \#DMR8603174 and \#DMR8903138 (G. Was). 


\section{REFERENCES}

[1] D.E. Alexander, G.S. Was and L.E. Rehn, submitted to J. Appl. Phys.

[2] H. A. Atwater, C.V. Thompson and H.I. Smith, Phys. Rev. Lett. 60 (2), 112 (1988).

[3] J. Li, J.C. Liu and J.W. Mayer, Nucl. Instrum. Methods B 36, 306 (1989).

[4] J. C. Liu, J. Li and J.W. Mayer, J. Appl. Phys. 67 (5), 2354 (1990).

[5] J.C. Liu, Thesis, Comell University, Ithaca, NY, 1989.

[6] L.E. Rehn, P.R. Okamoto, Nucl. Instrum. Methods B 39, 104 (1989).

[7] D.E. Alexander and G.S. Was manuscript in preparation.

[8] G.H. Vineyard, Radiat. Eff. 29, 245 (1976).

[9] P.G. Shewmon,Transformations in Metals (MicGraw-Hill, New York, 1969).

[10] H.V. Atkinson, Acta Metall. 36 (3), 469 (1988).

[11] M. Van Rossum and Y-T. Cheng, in Ion Implantation 1988, edited by F.H. Wohlbier (Trans Tech Publications Ltd., Sivitzerland, 1988) pp. 1-31. 

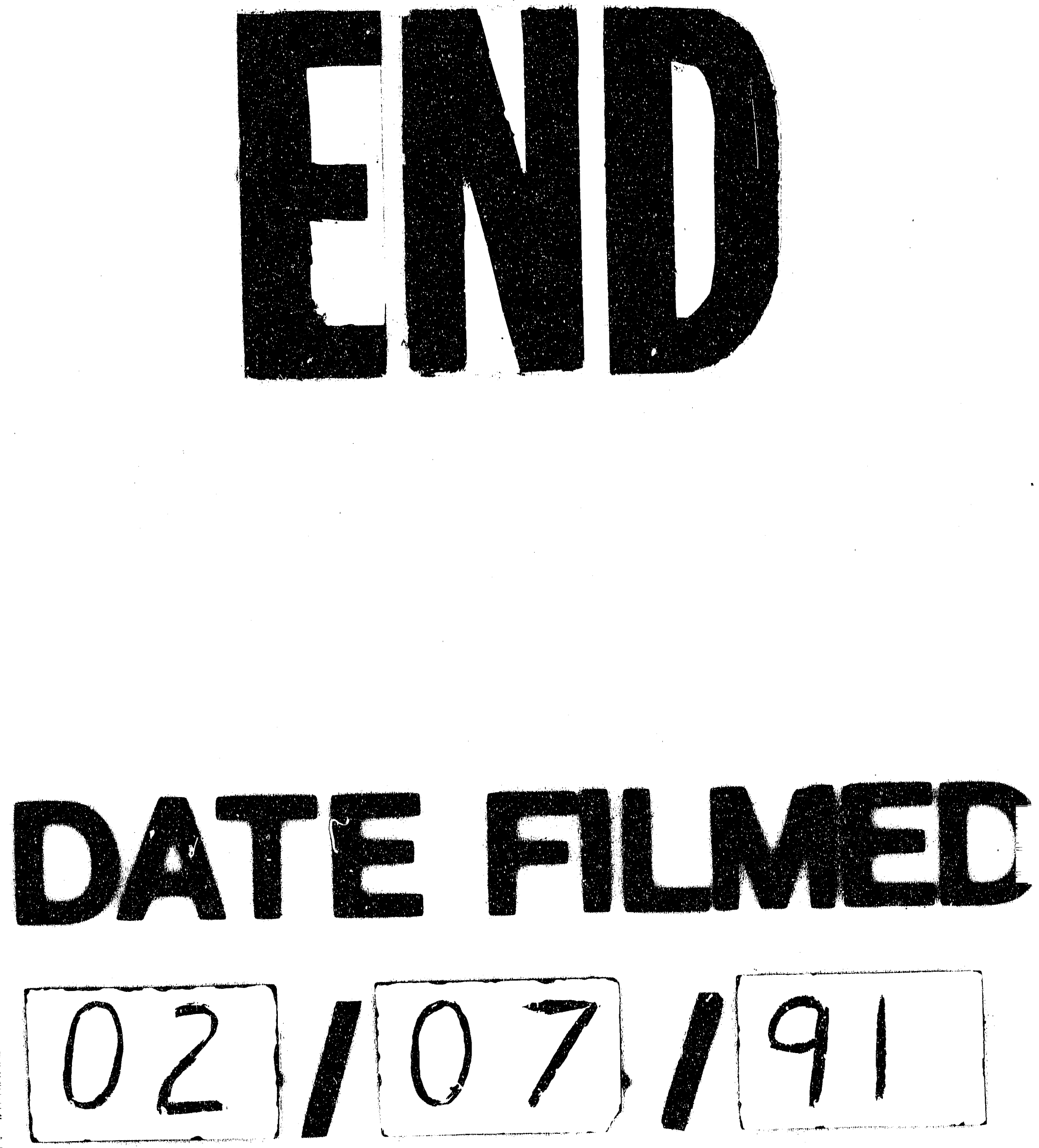


\section{mim}

